\title{
You Are Unique and So is Your Cancer-Targeting Cancer Precisely
}

\author{
Anit Jacob, Manas Ranjan Senapati, Niti Narang Raizada, S Varun Kumar and G Lohith* \\ Consultant Radiation Oncologist, Healthcare Global Enterprises Limited, Bangalore, India
}

*Corresponding author: G Lohith, Consultant Radiation Oncologist, Healthcare Global Enterprises Limited, Bangalore, India

\section{Introduction}

Cancer is a heterogeneous disease, meaning differences exist not only between cancer cells from various patients, but also between cancer cells within a single patient. Cancer is constantly evolving to evade death, and this is why no single drug has been effective in "curing" cancer. Usage of unique characteristics of each patient to focus, match and direct the most effective and precise treatments based not only on the genomic profiling of the patient and the cancer, but also on other unique characteristics distinguishing one patient from another is where the concept of precision oncology stems from. Every patient has a diverse and individualized genome, proteome, epigenome, micro-biome, lifestyle, diet, and other characteristics that interact to promote and influence cancer causation, disease progression, treatment options effectiveness, adverse events of drugs, remission, and relapse [1]. Emerging oncological paradigms encompassing precision immunotherapies, precision informatics, precisely targeted therapies, specific cellular engineering and artificial intelligence, based on nextgeneration sequencing, genomic profiling and supercomputing are increasingly combined and paired with traditional therapies like chemotherapy and radiation to address the many faces of cancer with high precision [2]. Precision oncology can be subdivided into precision diagnostics and precision therapy.

\section{Precision Diagnostics}

Diagnostics begin with an accurate and appropriate diagnosis of an individual patient and sub classifies population into cancer patients and individuals at high risk for specific cancers [3]. Establishing molecular classifications already existent in Colon cancers and breast cancers enhances the accuracy of therapeutic options [4]. The information derived from precision diagnosis reveals the precise medical measures, including surgery, radiation, chemotherapy, adjuvant therapy, that are needed for each individual [5].

\section{Radiomics and image-genomics}

Imaging in cancer such as ultrasound, computed tomography (CT), positron emission tomography (PET), or magnetic resonance imaging (MRI) is routinely used in diagnosis, staging, treatment planning, postoperative surveillance, and in response evaluation of cancer patients. Although these imaging modalities provide important phenotypic information of cancer, yet a great deal of genetic and prognostic information remains hidden [6]. Since imaging can be used to determine the three dimensions of the tumor, the concept of mapping the genomic heterogeneity could be evaluated by "radiomics," a field of study in which high-throughput data is extracted and large amounts of advanced quantitative imaging features are analyzed from medical images, and "imaging genomics," the field of study of high-throughput methods of associating imaging features with genomic data. The hidden information embedded in medical images may become utilized through these robust approaches and help in quantifying overall tumor spatial complexity and identifying the tumor sub-regions that drive disease transformation, progression, and drug resistance [7-9].

\section{Biomarkers and genetic tests}

"OMICS" based diagnosis can help oncologists revealing important genetic mutations that drive carcinogenesis. Technological advances such as next generation sequencing have clearly resulted in more effective therapeutic decisions and help in optimization of clinical outcomes, avoidance of unnecessary therapies, minimized side effects, and overcoming or avoiding drug resistance. Immunohistochemistry (IHC) and Genomic Profiling advances have helped to identify the presence of specific biomarkers and to risk stratify patients. Detection of human epidermal growth factor receptor 2 (HER2) in breast cancer and PDL1 in lung cancer as biomarkers have acted as targets for suitable drugs $[10,11]$. Mamma Print the 70-gene signature test for breast cancer has been evaluated in 6693 women with early-stage breast cancer to evaluate if the gene signature test could reduce the use of chemotherapy. Patients with high clinical risk and low genomic risk for recurrence, the difference in the 5-year survival rates between chemotherapy (98.8\%) and no-chemotherapy (97.3\%) was only $1.5 \%$ [12]. Approximately $46 \%$ of women with breast cancer at high 
clinical risk might not require chemotherapy and that the 70-gene signature could aid in treatment decisions, similarly Oncotype Dx generates a recurrence score (0-100) by analyzing the expression of 21 genes. Survival rates of patients with high recurrence scores (31 and higher) have been improved by adjuvant chemotherapy, whereas patients with low recurrence scores are unlikely to get benefits from the chemotherapy $[13,14]$.

\section{Liquid biopsies in precision medicine}

Predicting, diagnosing and monitoring tumors through noninvasive methods represent an important paradigm shift in precision medicine. Liquid biopsy is the analysis of circulating tumor cells (CTCs) and cell-free circulating nucleic acids, circulating tumor DNA (ctDNA) and exosomes) released in the peripheral blood from the primary tumor and/or metastatic deposits. A liquid biopsy or a simple blood sample can provide the genetic landscape and epigenetic characteristics of every cancerous lesion and offer the opportunity to dynamically track genetic and epigenetic changes. Treatment responses and resistance can be predicted. Details of the tumor genetic profile can enable the prediction of disease progression and response to therapies. Liquid biopsy can be used in cancer screening, patient stratification, and monitoring. In addition, short ctDNA fragments harbor the footprints of transcription factors, which could indicate the cell-type origin of pathological states, such as cancer [15]. However, knowledge regarding the physiology or biology of ctDNA, where it originates and how it moves into the circulation is sparse and obviously, more research is needed to answer these questions.

\section{Artificial intelligence in precision medicine}

Digital health, disruptive technologies such as biotechnology, wearable sensors, or artificial intelligence (AI) are gradually leading to three major directions

\section{a) Making patients the point-of-care}

b) Big huge data that require advanced analytics

c) Formulate the foundation of precision medicine

IBM Watson with its program provides clinicians with evidencebased treatment options. Watson for Oncology has an advanced ability to analyze structured and unstructured data in selecting a treatment pathway. Then by combining attributes from the patient's file with clinical expertise, external research, and data, the program identifies potential treatment plans for a patient [16]. Oncological development of pharmaceuticals and clinical trials take sometimes, more than a decade and costs billions of dollars. Speeding it up and making it more cost-effective would have an enormous effect on today's health care and how innovations reach everyday medicine. Artificial intelligence usage with supercomputers could root out therapies from a database of molecular structures such as the one tried for Ebola virus by Atom-wise Company [17].

\section{PDX MODELS}

PDX models use tumor fragments from cancer patients and are implanted into immune-deficient mice. Mice harboring the
PDX tumor fragment is tested and treated with multiple drugs to be tested and the efficacy of a drug can be predicted before being prescribed to the actual patient. Thus, PDX is a platform that provides evidence-based guidelines in choosing the correct and most effective drug to be prescribed to the specific cancer patient and important pathway for precision medicine [18]. Establishing PDX models worldwide reflects the high expectations of translating preclinical research to the clinic. EurOPDX launched cBio Portal, where information on models and their molecular annotation have been opened to the public to provide a platform of PDX studies [19].

\section{Precision Therapeutics}

Precision therapy matches the most effective treatment to the individual cancer patient based on the molecular profiling of the specific cancer and can be divided into two categories that include precision chemo-targeted therapy and precise therapeutic procedures. Precision chemo-targeted therapy is the use of the correct drugs prescribed that assures maximum benefit with minimum risk or toxicity for the patient. Precise Therapeutic measures could include surgery and radiation therapy tailored to the for individual patient's needs.

\section{Targeted therapy}

The most well-known and one of the earliest target-based treatments against breast cancer are directed at the HER2. Trastuzumab is a monoclonal antibody targeting HER2 and is used in patients with breast cancers overexpressing this receptor and inhibits the activity of HER2 expression. 469 women with metastatic breast cancer overexpressing HER2, standard chemotherapy with Trastuzumab attenuated disease progression compared to standard chemotherapy alone (median, 4.6 vs. 7.4 months). Survival time (median, 20.3 vs. 25.1 months) was also improved by the addition of trastuzumab to the chemotherapy [20]. Epidermal growth factor receptor (EGFR) inhibitor gefitinib has accelerated target-based therapy in lung cancer patients replacing cytotoxic chemotherapy for first-line therapy.(19). ALK is a cell surface protein that stimulates signaling pathways, such as the Ras/ Raf/MEK, PI3-K/mTOR, and Janus kinase (JAK)/signal transducer and activator of transcription (STAT) pathways and is activated by gene translocation and fusion with other genes. Crizotinib is an FDA-approved inhibitor of anaplastic lymphoma kinase (ALK), Ros proto-oncogene 1 (ROS1), and Met proto-oncogene (MET) [21].

Poly ADP-ribose polymerase (PARP) inhibitors are one of the most promising new classes of oncology drugs that target the DNA damage response and DNA repair. The PARP enzyme is important in the repair of single-stand DNA breaks and inhibitors of PARP prevent DNA repair, leading to cell death. These inhibitors are especially effective against cancer cells exhibiting gene mutations that are critical in the process of homologous recombination, a process that is crucial for repairing double-stranded DNA breaks. For example, the presence of the BRCA mutation and treatment with a PARP inhibitor, which blocks single strand DNA break repair, will lead to cell death. In a clinical trial, olaparib was beneficial against breast cancer [22]. 


\section{Immunotherapy}

A deeper understanding of tumor-immune interactions and development of immune checkpoint inhibitors has dramatically changed the therapeutic landscape of melanoma, NSCLCs and other malignancies. The immune system recognizes and is poised to eliminate cancer [23]. Pembrolizumab has been the most widely tested PD-1 inhibitor. In May 2017, it has been approved as firstline therapy in metastatic non-squamous NSCLC with combination of pemetrexed and carboplatin for patients with, independent of PD-L1 expression. Pembrolizumab has been approved for unresectable or metastatic tumor that expresses either microsatellite instability-high (MSI-H) or mismatch repair deficient biomarker [24]. Enthusiasm for immunotherapy has surged beyond targeting CTLA-4 and PD-1 pathways. Additional immune regulators, both stimulatory and inhibitory, are being explored as potential targets for cancer immunotherapy. Novel insights into neo-antigens and combination with immune checkpoint inhibition have breathed new life into cancer vaccines, which had been tested for decades with little success. Adoptive cell transfer of autologous $\mathrm{T}$ cells selected for tumor reactivity and genetically engineered $\mathrm{T}$ cells with chimeric antigen receptors targeting tumor antigens have demonstrated promise in melanoma and other cancer types. As new immunotherapies expand the arsenal of cancer therapies, deeper mechanistic insight will be required to inform clinical decisions. The exciting advances toward understanding and delivering precision immunotherapy are poised to change the way cancer is treated [25].

\section{Nanotechnology}

Utilization of nanotechnology has been tried for the development of new Nano-carrier systems that have the potential to provide an improved chemotherapeutic delivery through increased solubility and sustained retention. Targeted delivery of drug incorporated nanoparticles, through conjugation of tumorspecific cell surface markers, such as tumor-specific antibodies or ligands, which can enhance the efficacy of the anticancer drug and reduce the side effects. Additionally, multifunctional characteristics of the nano-carrier system would allow for simultaneous imaging of tumor mass, targeted drug delivery and monitoring (Theranostics) [26]. Nanoparticles can be engineered to avoid biological and biophysical barriers. Biological barriers might arise in the form of tight junctions between epithelial cells, as is the case for the blood-brain barrier (BBB), which impedes the extravasation of vascularly injected agents. Nanotechnology-based systems have shown efficacy in crossing the BBB by virtue of the properties of their constituent core materials. Endothelial vascular permeability might be increased by the co-administration of a bradykinin antagonist120. This indicates a strategy for the enhancement of EPR targeting of Nano vectors [27].

\section{CRISPR gene-editing system}

CRISPR DNA-cutting technique is used to delete programmed death-1 (PD-1) receptor and remove and replace defective T-cell receptors (TCR) with a new receptor that is engineered to sound the alarm against specific cancers (NYCE T-cells). Blood will be collected from patients and T-cells will be isolated and edited with
CRISPR before re-infusion back into the subjects. Basically, the study will convert normal human immune cells into cancer cell killers UCART019 is a type of CAR T-cell therapy that uses CRISPR to introduce a protein that enables T-cells to effectively recognize and kill cells expressing a protein called CD19, which is common in B-cell lymphoma and leukemia.. Two further clinical trials using CRISPR as a cancer immunotherapy also are ongoing in China. A Phase 2 clinical trial (NCT03081715) is investigating PD-1 knockout T-cells in an estimated 21 esophageal cancer patients. A Phase 1/2 trial (NCT03166878) is investigating UCART019 in up to 80 patients with B-cell lymphoma and leukemia [28].

\section{Radio sensitivity index: precision radiotherapy}

Torres-Roca et.al sought to identify genes correlated with RT response in 35 of the NCI-60 cell lines; they assessed the genetic contributions to radio sensitivity by quantifying the surviving fraction following exposure of the cell lines to a standard radiation dose of 2 Gy (the SF2 assay). [29]. Eschrich et.al subsequently expanded the model to 48 of the NCI-60 cell lines and included biological variables such as the mutation status of RAS and TP53, as well as tissue of origin. Using these data, a rank-based linear algorithm was created to calculate a radio-sensitivity index (RSI) [30]. The RSI has now been evaluated in independent cohorts spanning multiple tissue lineages [31]. The RSI has a demonstrated ability to predict for 5-year progression-free survival (PFS) and 5- year distant metastasis-free survival in two distinct NCI breast cancer database sets. The survival benefit was noted in patients treated with RT who were found to be radiosensitive (defined as an RSI above the $25^{\text {th }}$ percentile) compared with those who were found to be radio-resistant (RSI below the $25^{\text {th }}$ percentile). No difference in survival was noted between radiosensitive vs. radioresistant patients who were not treated with RT, further supporting the predictive value of the RSI as it relates to radiation treatment and helps in high precision and personalized step towards genomic based radiation dosing [32,33].

\section{Tumor treating fields in glioblastoma multiforme}

TT Fields is an antimitotic treatment modality that interferes with glioblastoma cell division and organelle assembly by delivering low intensity alternating electric fields to the tumor. Investigators delivered TT Fields treatment through 4 transducer arrays with 9 insulated electrodes each placed on the shaved scalp and connected to a portable device set to generate $200-\mathrm{kHz}$ electric fields within the brain. Adding tumor-treating fields (TT Fields) to temozolomide resulted in improved progression-free survival (PFS) and overall survival (OS) for patients with glioblastoma (GBM), according to a final analysis of results from the EF-14 trial. The addition of TT Fields did not lead to a significant increase in rates of systemic adverse events (AEs) compared with temozolomide monotherapy (48\% vs. $44 \%)$ [33,34].

\section{Conclusion}

Precision medicine requires a myriad of disruptive technologies to be implemented into developing treatments, practicing medicine, and delivering care. Because every single cancer patient exhibits a different genetic profile and the profile can change over time, more 
patients will benefit if therapeutic options can be tailored to that individual, thus avoiding the idea that "one-size-fits-all", Instead of applying a one-size-fits-all treatment, precision medicine's mantra is to provide the right treatment for the right patient at the right time.

\section{References}

1. Bode AM, Dong Z (2007) Precision oncology- the future of personalized cancer medicine? NPJ Precis Oncol 1(1): 2.

2. Bode, AM, DongZ (2018) Precision oncology- Recent advances in precision oncology research. npj Precision Oncology 2(11).

3. Friedman AA, Letai A, Fisher DE1, Flaherty KT (2015) Precision medicine for cancer with next-generation functional diagnostics. Nat Rev Cancer 15(12): 747-756.

4. Dienstmann R, Vermeulen L, Guinney J, Kopetz S, Tejpar S, et al. (2017) Consensus molecular subtypes and the evolution of precision medicine in colorectal cancer. Nat Rev Cancer 17(2): 79-92.

5. Cao Y (2017) EBV based cancer prevention and therapy in nasopharyngeal carcinoma. npj Precision Oncology 10.

6. Swanton C (2012) Intratumor heterogeneity: evolution through space and time. Cancer Res 72(19): 4875-4882.

7. Chong Y, Kim JH, Lee HY, Ahn YC, Lee KS, et al. (2014) Quantitative CT variables enabling response prediction in neoadjuvant therapy with EGFR-TKIs: are they different from those in neoadjuvant concurrent chemoradiotherapy?. PLoS One 9(2): 88598.

8. Divine MR, Katiyar P, Kohlhofer U, Quintanilla-Martinez L, Pichler BJ, et al. (2016) A population-based Gaussian mixture model incorporating 18F-FDG PET and diffusion-weighted MRI quantifies tumor tissue classes. J Nucl Med 57(3): 473-479.

9. Messiou C, Orton M, Ang JE, Collins DJ, Morgan VA, Mears D, et al. (2012) Advanced solid tumors treated with cediranib: comparison of dynamic contrast-enhanced MR imaging and CT as markers of vascular activity. Radiology 265: 426-436.

10. Moelans CB, Kibbelaar RE, van den Heuvel MC, Castigliego D, de Weger RA, et al. (2010) Validation of a fully automated HER2 staining kit in breast cancer. Cell Oncol 32(1-2): 149-155.

11. Rangachari D, VanderLaan PA, Shea M, Le X, Huberman MS, et al. (2017) Correlation between classic driver oncogene mutations in EGFR, ALK, or ROS1 and 22C3-PD-L1 > /=50\% Expression in lung adenocarcinoma. J Thorac Oncol 12(5): 878-883.

12. Fatima Cardoso, Laura J van't Veer, Jan Bogaerts, Leen Slaets, Giuseppe Viale, et al. (2016) 70-Gene signature as an aid to treatment decisions in early-stage breast cancer. N Engl J Med 375: 717-729.

13. Cronin M, Sangli C, Liu ML, Pho M, Dutta D, et al. (2007) Analytical validation of the oncotype DX genomic diagnostic test for recurrence prognosis and therapeutic response prediction in node-negative, estrogen receptor-positive breast cancer. Clin Chem 53(6): 10841091.

14. Sparano JA, Paik S (2008) Development of the 21-gene assay and its application in clinical practice and clinical trials. J Clin Oncol 26(5): 721-728.

15. Junyun Wang, Shuang Chang, Guochao Li, Yingli Sun (2017) Application of liquid biopsy in precision medicine: opportunities and challenges. Front Med 11(4): 522-527.
16. Shermer M (2017) Artificial intelligence is not a threat - yet. Sci Am Atom wise finds first evidence towards new Ebola treatments 316: 3-77.

17. Gao H (2015) High-throughput screening using patient-derived tumor xenografts to predict clinical trial drug response. Nat Med 21:13181325 .

18. Townsend EC (2016) The public repository of xenografts enables discovery and randomized phase II-like trials in mice. Cancer Cell 29: 574-586.

19. Slamon DJ, Leyland Jones B, Shak S, Fuchs H, Paton V, et al. (2001) Use of chemotherapy plus a monoclonal antibody against HER2 for metastatic breast cancer that overexpresses HER2. N Engl J Med 344(11): 783-792.

20. Ciardiello F, Tortora GA (2001) Novel approach in the treatment of cancer: targeting the epidermal growth factor receptor. Clin Cancer Res 7(10): 2958-2970.

21. Hallberg B, Palmer RH (2013) Mechanistic insight into ALK receptor tyrosine kinase in human cancer biology. Nat Rev Cancer 13(10): 685700 .

22. Robson M (2017) Olaparib for metastatic breast cancer in patients with a germline BRCA mutation. N Engl J Med 377: 523-533.

23. Topalian SL, Drake CG, Pardoll DM (2015) Immune checkpoint blockade: a common denominator approach to cancer therapy. Cancer Cell 27: 450-461.

24. Iwai Y, Hamanishi J, Chamoto K, Honjo T (2017) Cancer immunotherapies targeting the PD-1 signaling pathway. J Biomed Sci 24: 26.

25. Brentjens RJ (2011) Safety and persistence of adoptively transferred autologous CD19-targeted $\mathrm{T}$ cells in patients with relapsed or chemotherapy refractory B-cell leukemias. Blood 118: 4817-4828.

26. Shaker A Mousa (2017) Impact of nanobiotechnology on the future of medicine (nanomedicine): The road toward precision medicine. J Nanomed Nanotechnol 8(6).

27. Wu J, Akaka T, Maeda H (1998) Modulation of enhanced permeability in tumor by a bradykinine antagonist, a cyclooxygenase inhibitor. Cancer Res. 58: 159-165.

28. (2018) Immuno-oncology news. CRISPR Florida, USA.

29. Torres Roca JF, Eschrich S, Zhao H (2005) Prediction of radiation sensitivity using a gene expression classifier. Cancer Res 65: 7169-7176.

30. Eschrich S, Zhang H, Zhao H (2009) Systems biology modeling of the radiation sensitivity network: a biomarker discovery platform. Int J Radiat Oncol Biol Phys 75: 497-505.

31. Eschrich SA, Fulp WJ, Pawitan Y (2012) Validation of a radiosensitivity molecular signature in breast cancer. Clin Cancer Res 18: 5134-5143.

32. Eschrich SA, Pramana J, Zhang H (2009) A gene expression model of intrinsic tumor radio-sensitivity: prediction of response and prognosis after chemo-radiation. Int J Radiat Oncol Biol Phys 75: 489-496.

33. Mun EJ, Babiker HM, Weinberg U, Kirson ED, Von Hoff DD (2017) Tumor treating fields: a fourth modality in cancer treatment. Clin Cancer Res 24(2): 266-275.

34. Stupp R, Taillibert S, Kanner AA, Kesari S, Steinberg DM, et al. (2015) Maintenance therapy with tumor-treating fields plus temozolomide vs temozolomide alone for glioblastoma. Jama 314(23): 2535-2543. 
(c) 1 This work is licensed under Creative

To Submit Your Article Click Here: Submit Article

DOI: $10.32474 / \mathrm{SJO} .2019 .03 .000159$

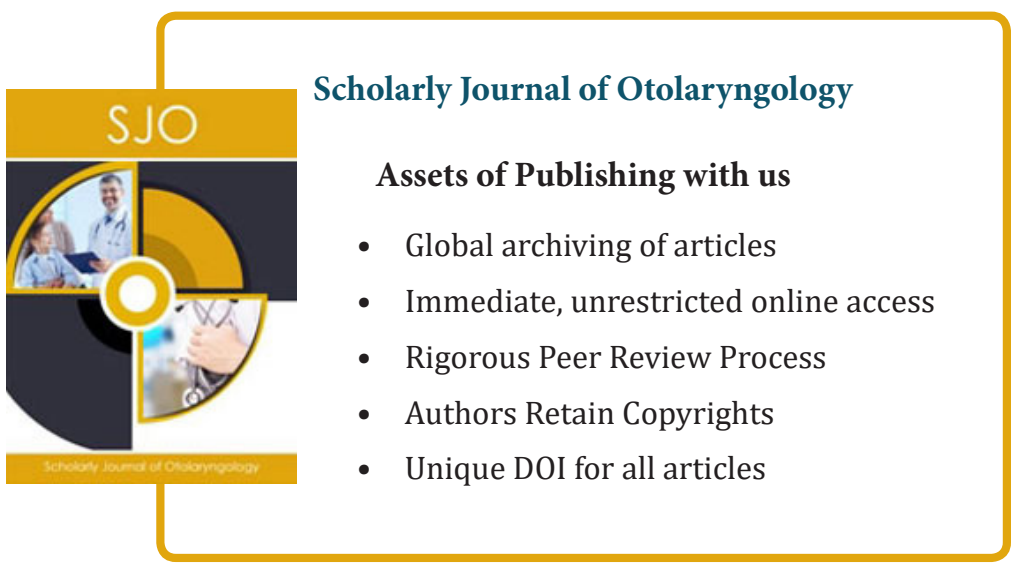

